\title{
A Novel Concept for Safe, Stiffness-Controllable Robot Links
}

\author{
Agostino Stilli, Helge A. Wurdemann, ${ }^{2}$ and Kaspar Althoefer ${ }^{3}$
}

\begin{abstract}
The recent decade has seen an astounding increase of interest and advancement in a new field of robotics, aimed at creating structures specifically for the safe interaction with humans. Softness, flexibility, and variable stiffness in robotics have been recognized as highly desirable characteristics for many applications. A number of solutions were proposed ranging from entirely soft robots (such as those composed mainly from soft materials such as silicone), via flexible continuum and snake-like robots, to rigid-link robots enhanced by joints that exhibit an elastic behavior either implemented in hardware or achieved purely by means of intelligent control. Although these are very good solutions paving the path to safe human-robot interaction, we propose here a new approach that focuses on creating stiffness controllability for the linkages between the robot joints. This article proposes a replacement for the traditionally rigid robot link - the new link is equipped with an additional capability of stiffness controllability. With this added feature, a robot can accurately carry out manipulation tasks (high stiffness), but can virtually instantaneously reduce its stiffness when a human is nearby or in contact with the robot. The key point of the invention described here is a robot link made of an airtight chamber formed by a soft and flexible, but high-strain resistant combination of a plastic mesh and silicone wall. Inflated with air to a high pressure, the mesh silicone chamber behaves like a rigid link; reducing the air pressure, softens the link and rendering the robot structure safe. This article investigates a number of link prototypes and shows the feasibility of the new concept. Stiffness tests have been performed, showing that a significant level of stiffness can be achieved - up to $40 \mathrm{~N}$ reaction force along the axial direction, for a $25-\mathrm{mm}$-diameter sample at $60 \mathrm{kPa}$, at an axial deformation of $5 \mathrm{~mm}$. The results confirm that this novel concept to linkages for robot manipulators exhibits the beamlike behavior of traditional rigid links when fully pressurized and significantly reduced stiffness at low pressure. The proposed concept has the potential to easily create safe robots, augmenting traditional robot designs.
\end{abstract}

Keywords: variable stiffness link, soft robotics, pneumatic actuation

\section{Introduction}

I $\mathrm{N}$ THE PAST DECADES, the introduction of soft robotics has redefined the limits of what a robot can accomplish. A number of soft, rubber or silicone-based manipulators have proved capable of achieving complex body poses, ${ }^{1}$ performing whole-body manipulation ${ }^{2}$ and exhibiting articulated movements using simple mechanical structures. ${ }^{3,4}$

Many silicone-based robotic systems make use of pneumatic actuation: a number of chambers independently actuated $^{5}$ or connected in a network ${ }^{6}$ are directly embedded in the silicone body of the robot. The controlled expansion or contraction of the pressurized chambers leads to a deforma- tion and thus movement of the overall structure. ${ }^{7}$ The main limitation of this type of actuation is the maximum acceptable deformation of the robot body and the maximum fluidic (pneumatic or hydraulic) pressure that its silicone structure is able to withstand without bulging or even blowing up.

As a consequence of these mechanical limitations, the maximum force exertable by such robotic systems is typically limited. ${ }^{1}$ A deformation of the same relative order of magnitude is produced in the legs of the walking soft robot proposed in an article ${ }^{7}$ once its pneumatic network is pressurized to perform a movement. These volume variations are generally difficult to model and thus difficult to predict, creating a significant control problem.

\footnotetext{
${ }^{1}$ Department of Informatics, Centre for Robotics Research, King's College London, London, United Kingdom.

${ }^{2}$ Department of Mechanical Engineering, University College London, London, United Kingdom.

${ }^{3}$ Faculty of Science \& Engineering, ARQ - Advanced Robotics at Queen Mary University of London, London, United Kingdom.
}

(C) Agostino Stilli et al. 2017; Published by Mary Ann Liebert, Inc. This is an Open Access article distributed under the terms of the Creative Commons Attribution License, which permits unrestricted use, distribution, and reproduction in any medium, provided the original work is properly cited. 
The STIFF-FLOP project (EU FP7-Grant No. 287728) attempted to overcome this issue developing bioinspired soft robotic surgical tools. ${ }^{8,9}$ The proposed modular manipulator in STIFF-FLOP was made out of silicone. Each module comprised three equally spaced pneumatically actuated chambers in parallel to the longitudinal axis. ${ }^{10}$ The STIFF-FLOP manipulator has embedded sensors such as F/T sensors and bending sensors to estimate the robot's tip pose. ${ }^{9,11-13}$ To limit the lateral inflation of the modules, each chamber was individually fiber reinforced, ${ }^{14}$ significantly improving the performance compared to externally braided robots. ${ }^{5}$ A similar approach has been investigated in an article, ${ }^{15}$ where the combination of different braiding angles was explored to achieve a desired bending behavior for the robot. The usage of external meshes for soft robotic fingers has been explored by researchers, as shown in Refs. ${ }^{16,17}$

To overcome the issue of large deformations associated with the pneumatic actuation of silicone-based robot manipulators, the authors of this article have introduced in Refs. ${ }^{18,19}$ a new type of soft robot, called the Inflatable Arm, composed of an inner silicone bladder with an outer nonextendable sleeve. The purpose of the sleeve is to further minimize undesired deformations of the soft body of the robot, constraining a deformable system into a nonextendable enclosure. Tendons attached to the outer sleeve at various locations allow the inflatable arm to be steered. It is noted that the robot uses a hybrid antagonistic actuation combining the advantages of pneumatic and tendondriven systems. Most importantly, the bioinspired arm is able to change its stiffness by concurrently regulating the chamber pressure and the extension of the tendons.

In this article, we propose and investigate a new type of silicone-based, stiffness-controllable, inflatable robotic link, using pneumatics. The work presented here follows the overall idea of creating inherently safe robots that can be used in modern industrial or surgical settings, attempting to provide solutions for safe human-robot interaction. In our envisaged solution, these inflatable links are the connecting elements between robot manipulator joints (possibly actuated by traditional electric motors, series elastic actuators, or even fluidic actuators), replacing common, rigid robotic links. This article describes the fabrication process and stiffness analysis of these novel links. Figure 1 shows the design and the working prin- ciple of the proposed link: a mesh is embedded in the lateral wall of a silicone cylinder, preventing it from expanding laterally and longitudinally once pressurized air is injected in the central chamber. We investigate the dependence between the design parameters of the mesh and the stiffness of the link.

This article is organized as follows: The design of the stiffness-controllable link and its fabrication process is presented in the Materials and Methods section. The investigation focused on the silicone materials and meshes to be used, to achieve adequate behavior for the main states of these links - soft and stiff. In the Experimental Setup and Results section, the experimental setup for stiffness evaluation is described and results presented. The Conclusions section summarizes the conclusions and achievements of this article and proposes future work and potential applications for the new concept.

\section{Materials and Methods}

The novel link is composed of two main structural elements: a plastic mesh embedded inside a layer of silicone in the shape of a hollow cylinder. When both ends of this cylindrical structure are closed, airtight chambers that can be pressurized are being achieved. Although its inflation is limited both in the longitudinal and radial direction by the inextensible mesh as shown in the force scheme of Figure 2, the mesh gives shape to the cylindrical link and prevents it from collapsing when it is in a low-pressure state. Exploring the influence on the stiffness of this type of link, a number of different silicone materials and plastic meshes were investigated at different pressure levels.

The proposed novel link is designed to be:

- stiffness controllable and inherently safe,

- easy to manufacture and inexpensive,

- made of soft material, but capable of behaving similar to a traditional rigid link,

- hollow, so to maximize the housing space inside the link body,

- lightweight, being made of a plastic mesh, silicone, and pressurized with air.

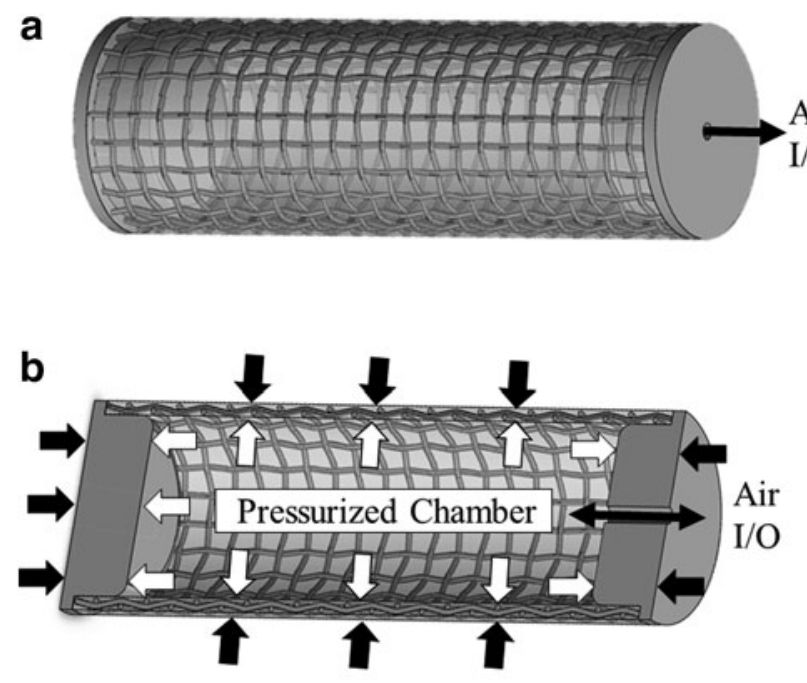

C

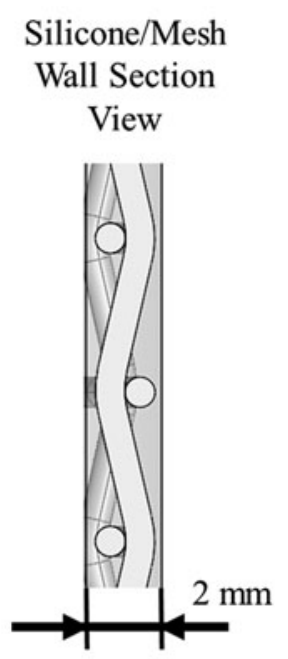

FIG. 1. CAD drawings of the stiffness-controllable link: overview of the link assembly (a), longitudinal section view of the link (b) showing the I/O channel for pressurized air, with white arrows showing the force distribution of the pressurized air inside the internal chamber and single-headed black arrows the force distribution of the reaction forces of the link. (c) Subfigure shows a longitudinal magnified section of the wall link, showing the mesh embedded in a $2 \mathrm{~mm}$ silicone layer. 
FIG. 2. Fabrication stages of the link: mesh sewn in the shape of a cylinder (a), mold components [I, II, and III (b)], and mold assembled (c). (d) Shows the link after demolding and removing of the silicone excess.

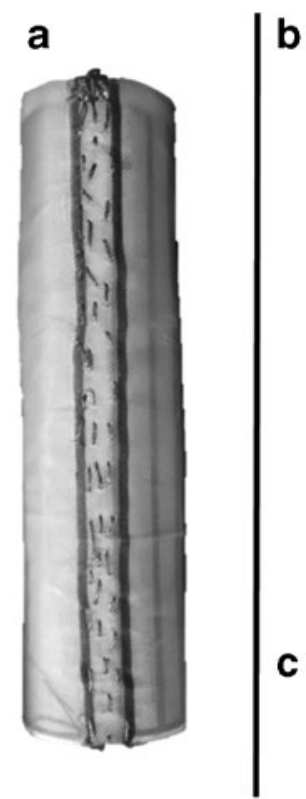

\section{Analysis of mesh and silicone properties}

To select a mesh suitable for this application, a number of parameters such as the shape and the aperture size of the patterns, as well as the material and diameter of the mesh threads, were considered.

Polypropylene (PP) mesh material is chosen as it is durable and does not react chemically with silicone-based materials. In addition, the surface of these meshes is smooth, and hence, the risk of perforation of the silicone membrane is considerably reduced. The low cost of these meshes allows the creation of inexpensive but robust and lightweight robotic links. Furthermore, any link diameter can be easily realized using the proposed fabrication process. A diamond-shaped aperture pattern was chosen for our application. This mesh aperture geometry was chosen among other commercially available meshes with different patterns due to its isotropic mechanical behavior and its negligibly low elasticity. These features match our requirements in terms of nondeformability of the link along longitudinal and radial directions.

Based on these considerations, four different diamond meshes from Normesh Ltd.* with different aperture sizes and thread diameters have been selected.

With regard to the selection of an appropriate silicone material, different silicone types (Dragon Skin ${ }^{\circledR}$, OOMOO $^{\circledR}$, and Ecoflex ${ }^{\circledR}$ ) were evaluated concerning their stiffness, chemical stability, and castability. Based on our analysis, we selected Dragon Skin, which proved to be most suitable exhibiting a good level of elasticity and shape retention. Aiming to achieve a good trade-off between softness of the material and mechanical rigidity of the cylindrical structure, Dragon Skin 20A was selected.

\section{Fabrication procedure stiffness-controllable link samples}

Concerning the meshes, we evaluated four PP meshes with aperture sizes between 105 and $420 \mu \mathrm{m}$, and corresponding thread diameters between 106 and $340 \mu \mathrm{m}$.

\footnotetext{
*Normesh Ltd.: www.normesh.co.uk/da/111262, accessed on
} June 30, 2015.
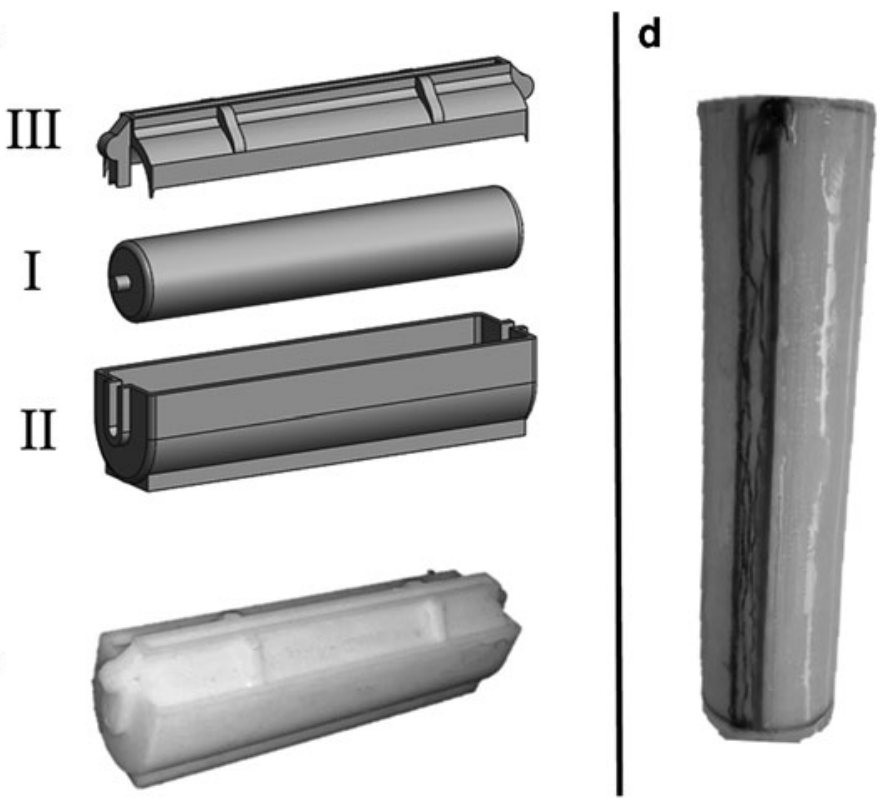

Rectangular sheets of the selected PP meshes of dimensions of $15 \times 8.5 \mathrm{~cm}$ were sewn in the shape of cylinders with a length of $15 \mathrm{~cm}$ and a diameter of $2.5 \mathrm{~cm}$ to create the link prototypes. Nylon threads were used to sew the longitudinal mesh sides together (with an overlap of $7 \mathrm{~mm}$ ) to form the cylindrical structures (Fig. 2a). The meshes were cut and sewn in a way that the main diagonal of the diamond apertures is aligned with the longitudinal axis of the link. A mold consisting of three components was created to allow each cylindrical mesh to be enclosed within the silicone-based material. The mold parts I, II, and III (Fig. 2b) were created by a Stratasys Dimension SST 768 rapid prototyping machine. The molding process is as follows: the sewn PP mesh is slipped over the cylindrical mold component I. Then, a silicone mixture is prepared and degassed in a vacuum chamber. Part of the silicone mixture is then poured into the base part of the mold (II), filling it for half of its capacity. Part I of the mold with the mesh is then inserted from the top into part II. More silicone is added after inserting part I into part II, to make sure that enough material is present to form a complete and continuous link wall. Part III is then inserted into part II as shown in Figure 2b; excess silicone escapes from the long aperture located at the top of part III. The silicone is then allowed to cure at room temperature for $8 \mathrm{~h}$. Figure 2c shows the mold in its assembled state. Figure $2 \mathrm{~d}$ shows the link after the molds and any excess silicone have been removed. The whole procedure was repeated to create four samples using the four different meshes.

Table 1 summarizes the characteristics of the samples, including the mesh aperture size, wall thickness, and thread diameter. The mold was designed on purpose in a way to generate silicone mesh walls of the same thickness for all four samples, regardless of the thickness of the used mesh.

\section{Experimental Setup and Results}

A test rig was prepared to evaluate the stiffness of the prototypes according to different levels of pressure applied. This investigation was aimed at understanding which mesh property impacted mostly on the overall stiffness of the system: the aperture size or the diameter of the thread used. 
Table 1. Variable Stiffness Link Samples, With Corresponding Mesh Aperture Sizes and Thread Diameter Embedded in Dragon Skin 20A Silicone

\begin{tabular}{lccc}
\hline Sample & $\begin{array}{c}\text { Aperture } \\
\text { size, } \mu m\end{array}$ & $\begin{array}{c}\text { Thread } \\
\text { diameter, } \mu m\end{array}$ & $\begin{array}{c}\text { Wall } \\
\text { thickness, mm }\end{array}$ \\
\hline (a) & 105 & 106 & 2 \\
(b) & 150 & 110 & 2 \\
(c) & 250 & 215 & 2 \\
(d) & 420 & 340 & 2 \\
\hline
\end{tabular}

To pressurize and test the samples, one of the two circular openings in the base of the cylinder was sealed using a customized 3D printed cap. The cap was inserted and secured in place with cable ties and silicone glue to guarantee the internal chamber to be air-tight. Figure 3a shows one of the samples ready for testing. After closing the tip with the cap, the open end of the sample was attached to a customized 3D printed test base. A pressure line is embedded in the base and connected to a pressure regulator (SMC ITV0010-3BS-Q). This allows the control of the pressure level inside the link. Figure 3 displays the experimental setup of the system for a lateral tip stiffness test. Cable ties and silicone glue were used on the base side as well as to ensure that the only air inlet/ outlet is via the pressure line. The pressure regulator was controlled by LabVIEW software. The level of stiffness of the samples was evaluated at the tip, where the displacement generated by an external applied force is maximal. Tests were performed pushing an ATI Nano17 Force/Torque sensor connected to a linear module toward the tip as shown in Figure 3-laterally along the $x$-axis and longitudinally at the front of the tip along the $z$-axis. To measure $F_{z}$, a linear slide was rotated counter-clockwise by $90^{\circ}$ in comparison with the pose shown in Figure 3, so that the linear module with the mounted F/T sensor exerts forces along the $z$-axis direction.

Both test scenarios have been performed applying four different pressure levels of 15, 30, 45, and $60 \mathrm{kPa}$. Each experiment started with the ATI Nano17 F/T sensor being $0.5 \mathrm{~mm}$ away from the silicone surface of the sample. The lateral stiffness tests consisted of load and unload cycles achieving a deflection of $15 \mathrm{~mm}$. For longitudinal stiffness tests, a smaller displacement of $5 \mathrm{~mm}$ was achieved so to avoid buckling phenomena. Each loading/unloading cycle was performed five times to compute the variance of the measurements. It is important to note that no significant radius or length variations occurred after pressurizing the samples, further proving the effectiveness of this design in terms of limitation of undesirable deformations of this silicone-based system.

\section{Stiffness evaluation-lateral tip test}

For each of the four link samples, the lateral tip test has been performed applying four different pressure levels. Each loading and unloading cycle was repeated five times. The results of these tests are shown in Figure 4 with the deflection displacement plotted against force.

In Figure 4, the average values of the five force reading cycles are plotted with the corresponding error bars for each pressure level in separate graphs. As shown in Figure 4a, the load-unload curves have an overall linear trend and some hysteresis. The maximum achievable force is $2.09 \mathrm{~N}$ at $15 \mathrm{kPa}$. Increasing the pressure to 30,45 , and $60 \mathrm{kPa}$, the force increases to $2.61,2.85$, and $3.01 \mathrm{~N}$, respectively. As expected, applying a larger pressure leads to an increase in stiffness of the inflated link and, thus, an increase of lateral reaction force. Comparing the graphs of Figure 5, showing the readings obtained from experiments with a different mesh-silicone combination, it can be deduced that the bigger the thread diameter and the aperture sizes are, the stiffer is the inflated link. For instance, the mesh used in Figure $5 \mathrm{a}$ at $60 \mathrm{kPa}$ exerts a lateral force of $3.01 \mathrm{~N}$, whereas the one used in Figure $5 \mathrm{~d}$ with the same level of internal pressure can exert more than double the amount of reaction force at $6.55 \mathrm{~N}$, with the same displacement.

\section{Stiffness evaluation-longitudinal tip tests}

The results of the stiffness tests for the four links along the longitudinal direction on the tip are shown in Figure 5a-d. Each graph shows force values against tip displacement, calculated as the average of five load-unload cycles for four different pressure levels. Error bars are included.

Comparing Figures 4 and 5, it is clear that the forces measured at a displacement of $5 \mathrm{~mm}$ for the longitudinal tip stiffness tests
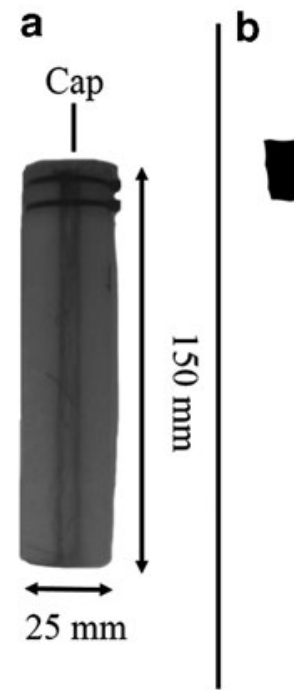
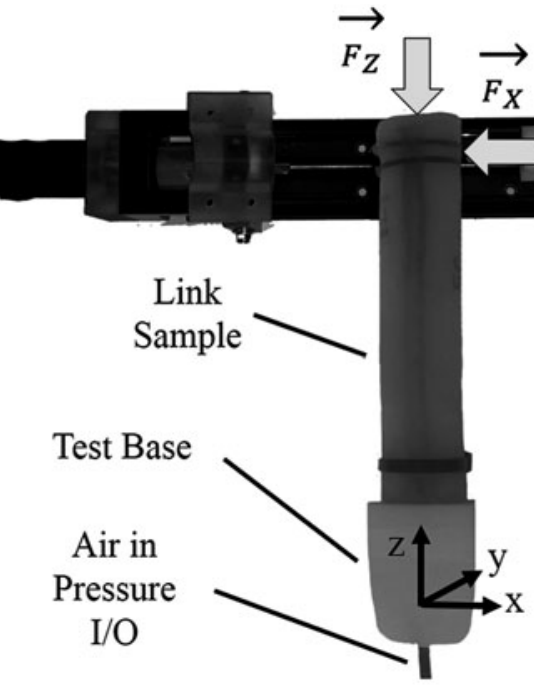

FIG. 3. Link sample with 3D printed cap embedded for stiffness test (a) and setup for the lateral stiffness tests at the tip (b) direction of the lateral force Fx and of the longitudinal force $\mathrm{Fz}$ applied during the tests are marked. 

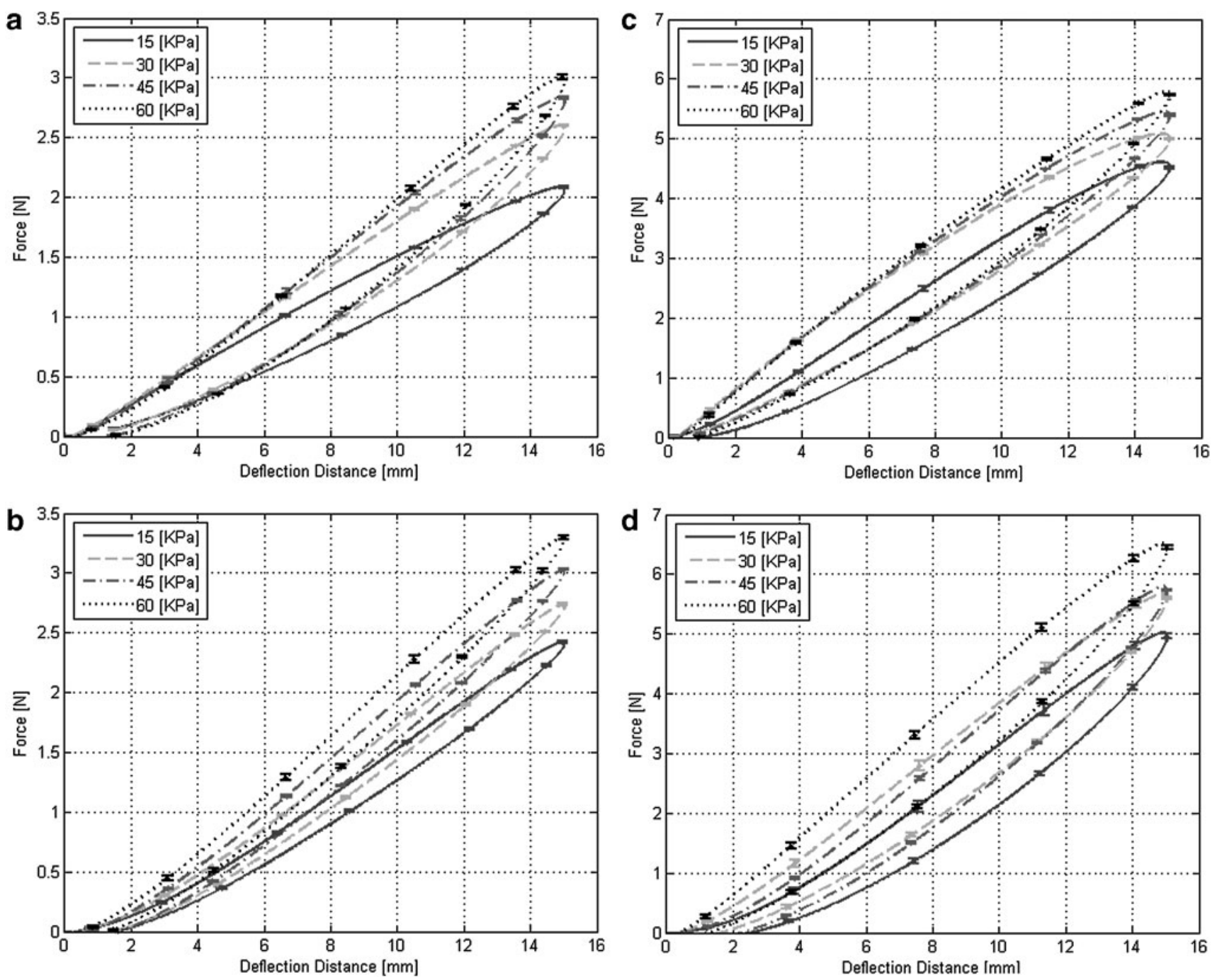

FIG. 4. Deflection distance versus force at the link tip laterally applied for samples (a), (b), (c), and (d) at 15, 30, 45, and $60 \mathrm{kPa}$. Standard deviation values ordered by increasing pressure level: (a) $0.007,0.006,0.0144,0.009 ;$ (b) $0.009,0.008$, $0.008,0.018 ;$ (c) $0.018,0.016,0.013,0.016 ;$ (d) $0.041,0.039,0.017,0.036$.

exceeded consistently the corresponding measurements of the lateral stiffness tests. For instance, the link shown in Figure 5a pressurized at $15 \mathrm{kPa}$ produces a maximum reaction force of $2.09 \mathrm{~N}$, if stressed laterally, with a displacement of $15 \mathrm{~mm}$; however, the same link sample leads to a peak reaction force of $11.90 \mathrm{~N}$ and an associated displacement of $4.2 \mathrm{~mm}$ only, when the force is applied longitudinally (Fig. 5a). Thus, the sample behaves in a similar manner to a rigid beam, particularly one with a circular section. The mechanical properties of this soft robotic link structure make the modeling significantly simpler when compared to the typical silicone-based robot manipulators. It is important to note that the peak force values during a loadunload cycle are reached for the longitudinal tests before the displacement of $5 \mathrm{~mm}$ is reached. Once again, this can be explained considering the system as a cantilever beam (clamped on one base, free at the other end). The compression force along the longitudinal axis produces buckling behavior after a deformation of $3.5-4.5 \mathrm{~mm}$; the beginning of the buckling corresponds to the trend inversion in the exerted force. Also in this case, the obtained force readings for the four prototypes display a continuous increase considering the increasing pressure levels as shown in
Figure 5. Furthermore, the link prototypes composed of meshes with bigger threads and aperture sizes exhibit a stiffer behavior. Table 2 summarizes the experimental results.

\section{Conclusions}

Departing from the traditional approach to robot arms with their rigid links, this article proposes a novel concept for stiffness-controllable robot links. The proposed concept is inspired by our earlier work on inflatable, stiffness-controllable robotic manipulators. ${ }^{18}$

The proposed concept provides a new solution to vary the stiffness of robot manipulator links with ramifications especially in the area of safe human-robot interaction (HRI). In contrast to current solutions for safe HRI using stiffnesscontrollable joints such as those based on variable elastic actuators, we pursue a different approach here where the links are the focus of our attention and whose stiffness can be controlled to adapt to the environmental situations, especially to render the robot safe when, for example, a human is in the nearby vicinity, by rapidly reducing the stiffness. 

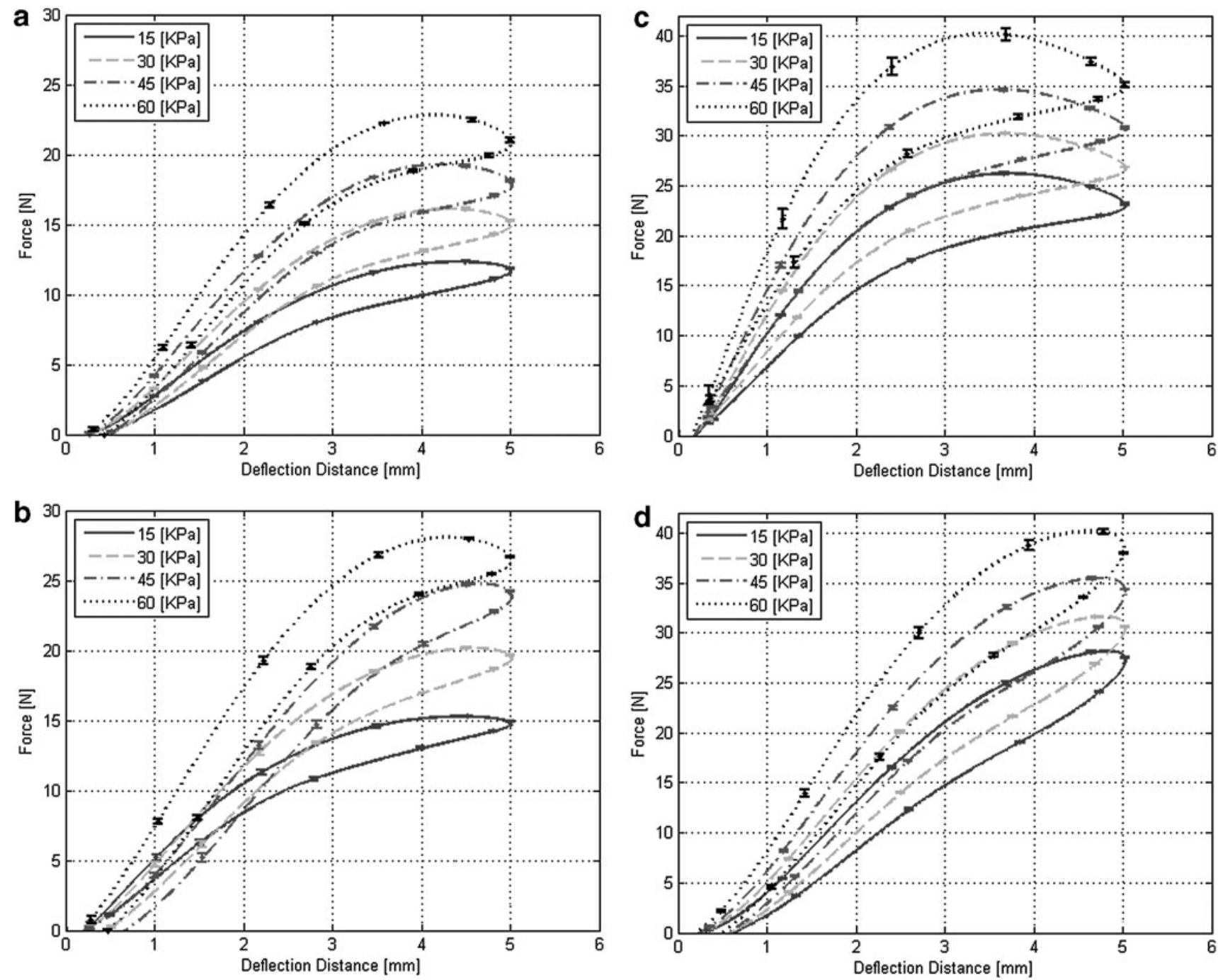

FIG. 5. Deflection versus force at the link tip longitudinally applied for samples (a), (b), (c), and (d) at 15, 30, 45, and $60 \mathrm{kPa}$. Standard deviation values ordered by increasing pressure level: (a) $0.051,0.080,0.089,0.153 ;$ (b) $0.150,0.136$, $0.178,0.179$; (c) $0.133,0.123,0.206,0.536$; (d) $0.095,0.100,0.121,0.255$.

Table 2. Comparison of Peak Force Values at Different Pressure Levels for the Four Samples Analyzed in the Lateral and Longitudinal Stiffness Test

\begin{tabular}{|c|c|c|c|c|c|c|}
\hline & & & \multicolumn{4}{|c|}{ Peak forces [N] } \\
\hline \multicolumn{2}{|c|}{ Stiffness test } & Sample & $15 \mathrm{kPa}$ & $30 \mathrm{kPa}$ & $45 \mathrm{kPa}$ & $60 \mathrm{kPa}$ \\
\hline \multirow{4}{*}{ 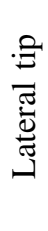 } & \multirow{4}{*}{ 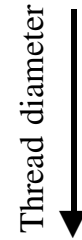 } & (a) & 2.09 & 2.61 & 2.85 & 3.01 \\
\hline & & (b) & 2.42 & 2.73 & 3.03 & 3.30 \\
\hline & & (c) & 4.55 & 5.00 & 5.40 & 5.83 \\
\hline & & (d) & 5.04 & 5.71 & 5.81 & 6.55 \\
\hline \multirow{4}{*}{ 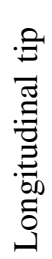 } & \multirow{4}{*}{ 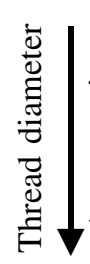 } & (a) & 11.90 & 15.10 & 17.90 & 20.90 \\
\hline & & (b) & 14.90 & 19.50 & 23.70 & 26.50 \\
\hline & & (c) & 23.50 & 27.20 & 31.50 & 35.80 \\
\hline & & (d) & 27.80 & 31.10 & 34.70 & 39.10 \\
\hline
\end{tabular}

Shaded gradients show force trends in relation to pressure level and mesh geometrical characteristics. 
As presented in this article, we propose our stiffnesscontrollable link concept as a replacement of the rigid links of traditional robot arms. We believe that our approach is advantageous because we consider turning a standard joint into one with compliant features a more complicated approach. One can, however, conceive a robot arm design, which combines our stiffness-controllable links with joints whose compliance is also controllable, if required. Additional advantages of the proposed concept are as follows:

- high rigidity with low deformation when pressurized,

- light-weight, low-inertia structure,

- ample space inside the inflatable link that can be used for cabling,

- opportunity to estimate interaction forces based on measured pressure within the inflatable links.

The last point is particularly useful in the context of safety, since a controller with pressure feedback can be easily implemented to depressurize the manipulator links when an impact is detected, to cushion a collision with a human appropriately, considerably reducing the risk of injury. Hence, the proposed link concept is especially suitable for applications that involve HRI and collaboration.

\section{Acknowledgments}

This research has received funding from the European Commission's Seventh Framework Programme, project STIFF-FLOP under grant agreement no. 287728, and from Horizon 2020 Research and Innovation Programme, project FourByThree under grant agreement no. 637095. The authors acknowledge the King's College London, Centre for Robotic Research for the support of the experimental studies.

\section{Author Disclosure Statement}

No competing financial interests exist.

\section{References}

1. Laschi C, Cianchetti M, Mazzolai B, Margheri L, Follador M, Dario P. Soft Robot Arm Inspired by the Octopus. Adv Robot 2012;26:709-727.

2. Martinez RV, Branch JL, Fish CR, Jin L, Shepherd RF, Nunes $\mathrm{RMD}$, et al. Robotic tentacles with three-dimensional mobility based on flexible elastomers. Adv Mater 2013;25:205-212.

3. Lin H-T, Leisk GG, Trimmer B. GoQBot: a caterpillarinspired soft-bodied rolling robot. Bioinspir Biomim 2011; 6:26007.

4. Suzumori K, Endo S, Kanda T, Kato N, Suzuki H. A bending pneumatic rubber actuator realizing soft-bodied manta swimming robot. In IEEE International Conference on Robotics and Automation; Rome: IEEE, 2007, pp. 4975-4980.

5. Cianchetti M, Ranzani T, Gerboni G, De Falco I, Laschi C, Menciassi A. STIFF-FLOP surgical manipulator: mechanical design and experimental characterization of the single module. In IEEE/RSJ International Conference on Intelligent Robots and Systems; Tokyo: IEEE, 2013, pp. 3576-3581.

6. Mosadegh B, Polygerinos P, Keplinger C, Wennstedt S, Shepherd RF, Gupta U, et al. Pneumatic networks for soft robotics that actuate rapidly. Adv Funct Mater 2014;24:2163-2170.

7. Shepherd RF, Ilievski F, Choi W, Morin SA, Stokes AA, Mazzeo AD, et al. Multigait soft robot. Proc Natl Acad Sci U S A 2011;108:20400-20403.
8. Noh Y, Sareh S, Back J, Wü HA, Ranzani T, Lindo E, et al. A three-axial body force sensor for flexible manipulators. In 2014 IEEE International Conference on Robotics and Automation (ICRA); IEEE: pp. 6388-6393.

9. Sareh S, Jiang A, Faragasso A, Noh Y, Nanayakkara T, Dasgupta P, et al. Bio-inspired tactile sensor sleeve for surgical soft manipulators. In IEEE International Conference on Robotics and Automation (ICRA); Hong Kong: IEEE, 2014, pp. $1454-1459$.

10. Ranzani T, Cianchetti M, Gerboni G, De Falco I, Petroni G, Menciassi A. A modular soft manipulator with variable stiffness. In 3rd Joint Workshop on New Technologies for Computer/Robot Assisted Surgery: Verona, 2013, pp. 11-13.

11. Wurdemann HA, Sareh S, Shafti A, Noh Y, Faragasso A, Chathuranga DS, et al. Embedded electro-conductive yarn for shape sensing of soft robotic manipulators. In Engineering in Medicine and Biology Society (EMBC), 2015 37th Annual International Conference of the IEEE; Milan: IEEE, 2015, pp. 8026-8029.

12. Noh Y, indo Secco EL, Sareh S, Wurdemann H, Faragasso A, Back J, et al. A continuum body force sensor designed for flexible surgical robotics devices. Conf Proc IEEE Eng Med Biol Soc 2014;2014:3711-3714.

13. Noh Y, Sareh S, Wurdemann H, Liu H, Back J, Housden J, et al. Three-axis fiber-optic body force sensor for flexible manipulators. IEEE Sens J 2016;16:1641-1651.

14. Fras J, Czarnowski J, Macias M, Glowka J, Cianchetti M, Menciassi A. New STIFF-FLOP module construction idea for improved actuation and sensing. In IEEE International Conference on Robotics and Automation (ICRA); Seattle: IEEE, 2015, pp. 2901-2906.

15. Faudzi AAM, Razif MRM, Nordin INAM, Suzumori K, Wakimoto S, Hirooka D. Development of bending soft actuator with different braided angles. In IEEE/ASME International Conference on Advanced Intelligent Mechatronics, AIM 2012; Kaohsiung, Taiwan: IEEE, pp. 1093-1098.

16. Deimel R, Brock O. A novel type of compliant and underactuated robotic hand for dexterous grasping. The International Journal of Robotics Research; 2015: p.0278364915592961.

17. Wall V, Deimel R, Brock O. Selective stiffening of soft actuators based on jamming. In 2015 IEEE International Conference on Robotics and Automation (ICRA); Seattle: IEEE, 2015, pp. 252-257.

18. Stilli A, Wurdemann HA, Althoefer K. Shrinkable, stiffnesscontrollable soft manipulator based on a bio-inspired antagonistic actuation principle. 2014 IEEE/RSJ International Conference on Intelligent Robots and Systems (IROS 2014); Chicago: IEEE, 2014, pp. 2476-2481.

19. Maghooa F, Stilli A, Noh Y, Althoefer K, Wurdemann HA. Tendon and pressure actuation for a bio-inspired manipulator based on an antagonistic principle. In 2015 IEEE International Conference on Robotics and Automation (ICRA); Seattle: IEEE, 2015, pp. 2556-2561.

Address correspondence to: Kaspar Althoefer ARQ-Advanced Robotics at Queen Mary Faculty of Science \& Engineering Queen Mary University of London Mile End Road London E1 4NS United Kingdom

E-mail: k.althoefer@qmul.ac.uk 